\title{
天基信息实时智能服务系统仿真评估研究
}

\author{
江昊 ${ }^{1,2}$, 李德仁 ${ }^{1,3}$, 沈欣 ${ }^{1,3}$, 吴静 ${ }^{1,2}$ \\ （1. 地球空间信息技术协同创新中心，武汉 430079；2. 武汉大学电子信息学院，武汉 430072; \\ 3. 武汉大学测绘遥感信息工程国家重点实验室, 武汉 430079)
}

\begin{abstract}
摘要: 天基信息实时智能服务系统（PNTRC）是多星协同、多网互联的下一代天基信息系统，将广泛应用在卫星通信、导航、 遥感等领域。本文针对 PNTRC 建模仿真和效能评估展开研究, 分析了系统评估仿真的必要性、需求以及发展趋势, 进而支 撑 PNTRC 顶层设计和实施方案论证。针对 PNTRC 动态时变、业务多样、异质异构的特性，对场景可扩展的高效分布式仿真、 时空动态分析评估方法、系统体系化建模等关键技术展开详细讨论。基于分布式仿真评估系统, 以高分辨率对地遥感业务为 例, 对比和评估了多个低轨通信星座对遥感业务的传输支持能力。研究结果表明, 在遥感业务数据传输过程中, 覆盖密集的 OneWeb 星座卫星具有更小的网络时延、丢包以及更高的容量和稳定性，但单星容量反而不及覆盖密度较小的 “虹云” “鸿雁” 星座。本文研究为我国 PNTRC 可行性论证与工程建设提供了方法和技术手段支撑。
\end{abstract}

关键词: 天基信息；实时服务；仿真；评估；低轨通信星座

中图分类号：TP391.9 文献标识码：A

\section{Simulation and Evaluation of Real-Time and Intelligent Space-Based Information Service System of China}

\author{
Jiang Hao ${ }^{1,2}$, Li Deren ${ }^{1,3}$, Shen Xin ${ }^{1,3}$, Wu Jing ${ }^{1,2}$ \\ (1. Collaborative Innovation Center of Geospatial Technology, Wuhan 430079, China; 2. Electronic Information School, \\ Wuhan University, Wuhan 430072, China; 3. State Key Laboratory of Information Engineering in \\ Surveying, Mapping and Remote Sensing, Wuhan University, Wuhan 430079, China)
}

\begin{abstract}
The real-time and intelligent space-based information service system is a next-generation space-based information system featuring multi-satellite coordination and multi-network interconnection. It will be widely used in satellite communications, navigation, and remote sensing. To support the top-level design and construction of the real-time and intelligent space-based information service system, this paper studies the modeling simulation and performance evaluation of the system, and analyzes the necessity, requirements, and development trends of system simulation and evaluation. The key technologies such as scalable and efficient distributed simulation, spatial-temporal dynamic evaluation, and systematic modeling are discussed, considering the characteristics of the system, such as dynamic time-varying, diverse business, and heterogeneity. Based on the design of the distributed simulation and evaluation system, the transmission support capabilities of different low-orbit communication satellite systems are compared and evaluated, taking high-resolution remote sensing as an example. The results show that during the remote sensing data transmission process, the OneWeb satellite constellation has smaller delay, less packet loss, and higher capacity and stability, owing to its dense coverage; however, its capacity per satellite is inferior to that of the Hongyun satellite constellation which has a smaller coverage density. This study can provide technical supports for the feasibility evaluation and construction of the real-time and intelligent space-based information service system.
\end{abstract}

Keywords: space-based information; real-time services; simulation; evaluation; low-orbit communication satellite system

收稿日期 : 2019-12-04; 修回日期 : 2020-02-28

通讯作者 : 江昊, 武汉大学电子信息学院教授, 研究方向为通信与信息系统、空间信息网络; E-mail: jh@whu.edu.cn

资助项目：中国工程院咨询项目 “天基信息实时服务系统（PNTRC）发展战略研究” (2017-ZD-01)

本刊网址： www.engineering.org.cn/ch/journal/sscae 


\section{一、前言}

建设天基信息实时智能服务系统 (PNTRC), 提供高性能的导航、定位、授时、遥感、通信一 体化实时服务 [1,2], 可化解中国北斗卫星导航系 统 (简称北斗系统) 海外建设地基增强站受限难题, 抢占通信 / 导航 / 遥感集成服务这一新兴科技制高 点, 也是实现我国在空间科技领域 “弯道超车”, 带 动卫星遥感、卫星通信、卫星导航等关联产业创新 发展的重要推动力。

我国空天信息的响应速度、覆盖范围与世界领 先水平差距明显 [3 9]。以美国为例, 依托空天信 息网络, 在全球范围内从发现目标到遏制目标的响 应周期约为 $10 \mathrm{~min}$ 。相比之下，我国尚未完全建成 空天网络链路, 从发现敏感事件到信息传输与处理, 再到决策反应, 对于全球事件有效响应的周期超过 $10 \mathrm{~h}$ 。PNTRC 的建设和应用, 将显著改善响应速度 和覆盖范围, 有望接近甚至部分达到国际先进水平。 预计到 2035 年, 我国将发射超过 200 颗的智能遥 感与导航增强卫星，与现有和规划中的天基系统深 度融合，建成全球服务系统，掌握全球天基制信息 权，形成天基信息大众化、商业化应用并覆盖全球 范围的新局面。

当前, 世界航天大国将重点竞争方向转向低轨 通信卫星和遥感卫星星座，纷纷投入巨额资源予以 快速发展; 但尚未提出通信/导航/遥感一体化的 空天信息服务系统概念或方案, 相关建设经验也属 空白。我国 PNTRC 具有前瞻性, 未来系统的建设 和运行方案面临着技术挑战。因此, 在相关方案的 论证过程中、启动建设前, 需要针对可行性、整体 效能等开展详尽的性能评估和虚拟验证, 而系统仿 真是应对这一重要需求的有效工具。

研究 PNTRC 的综合效能, 需要将我国现有各 类通信卫星、导航卫星、遥感卫星和其他空间平台 进行组网，通过协同观测和信息中继来实现对各类 任务协同的灵活性。由于缺少已有系统的参照, 无 法直观确定具有最优应用效能的网络结构, 需要借 助系统仿真结果来逐步探索, 通过多种候选方案的 性能对比来寻优。本文针对 PNTRC 建设的方案论 证需求, 进行大量的仿真架构设计和数值实验, 依 据评估结果来分析各种建设方案的优劣势, 从而给
出系统核心技术突破方面的方向性建议。相关仿真 评估方法可为 PNTRC 架构优化和深化研究提供基 础性支撑。

\section{二、空间信息系统仿真评估研究现状}

天基信息实时智能服务作为空间信息网络的重 要呈现形式, 是由若干颗（多于 100 颗）搭载高分 辨率可见光、红外或合成孔径雷达 (SAR) 等遥 感载荷以及导航增强载荷的低轨卫星组成的天基 系统。鉴于空间信息网络的大规模、高动态、快 速时变等特性, 常用的空间网络仿真模型一般具 有较高的抽象程度, 较多针对特定应用或特定通 信骨干、在单一仿真软件上进行分析研究 [10]。 然而, 具有可扩展能力的系统级仿真技术是目前 空间信息网络建设的迫切需求。只有在系统级仿 真的基础上，才能进一步对天基信息实时智能服 务的各子系统、未来可扩展业务、整体效能等进 行多层次和全方位的评估。目前, 在大规模系统 仿真方面多采用高层体系结构 (HLA) 、运行支撑 环境 (RTI)、数据分发服务 (DDS) 等分布式仿 真服务框架 [11,12]。可扩展性和分布式仿真系统 架构是系统仿真发展的必然趋势, 但针对空间信 息网络的节点对象、拓扑特征、信息流特性进行 适应性的研究工作还比较少。

天基信息实时智能服务的目标是支撑定位、导 航、授时、遥感、通信信息服务，而各类业务和应 用都有不同的指标体系。(1)在通信业务方面, 鲁娜 等 [13] 针对卫星通信系统的抗干扰性能评估, 提出 了由抗电磁干扰性能和抗摧毁威胁性能构成的指标 体系; 许相莉等 [14] 针对作战领域的卫星通信系统 的功能和性能, 提出物理域、信息域、认知域和社 会域 “四域” 范畴下的指标体系。(2)在遥感业务 方面, 彭耿 [15] 针对遥感卫星系统信息支援能力 问题，提出了面向具体任务有效时长、并与用户应 用 (任务) 紧密耦合的能力评估指标体系; 覃鹏程 等 [16] 针对遥感卫星应用体系贡献度问题, 设计了 基于任务需求的体系贡献度评估模型。(3)在导航定 位授时业务方面, 段亚军等 [17] 依据反映卫星导航 系统主要功能的指标, 构建系统综合效能评估模型; 郭树人等 [18] 提出, 卫星导航系统服务性能评估指 
标体系应当重点考虑空间与时间基准、导航信号等 核心指标，据此设计了 “北斗三号” 系统的服务性 能规范。

天基信息实时智能服务具有时变特性, 能够调 度多项功能来协同完成单一任务, 而单一的指标体 系和静态的评估方法将无法对如此复杂的网络系统 进行效能评估。因此, 类似天基信息实时智能服务 这样的空间信息网络系统仿真, 朝着指标体系层次 化、时空评估动态化的趋势发展。天基信息实时智 能服务的异构异质性强, 承载业务信息流与通信网 络之间存在多层次交错耦合的关系，单一、确定性 模型很难描述各类业务流的特性以及业务流、网络 特性、用户体验质量间的关系。耦合各类网络因素, 对天基信息实时智能服务进行体系化建模, 才能透 彻分析网络时空特性成因, 进而实施准确评估和优 化 $[19,20]$ 。

\section{三、PNTRC 仿真评估总体分析}

PNTRC 星座由数量众多的卫星构成, 开展仿 真评估研究, 不仅需要针对单颗卫星, 还需要对 系统整体进行评估。PNTRC 服务能力按照功能不 同分为三部分: 通信服务能力、遥感服务能力、 导航定位授时服务能力, 而整体服务能力是具有 综合意义的指标。

\section{（一）通信仿真评估}

PNTRC 涉及低轨通信卫星、遥感卫星、导航 卫星、地面网络等多种网络, 具有高动态网络拓扑、 信息流多样、节点异构等特征。经综合分析, 可将 PNTRC 抽象为多层空间信息时效网络模型, 进而 从多个角度开展通信能力分析。

实际应用的各类通信卫星, 所具有的通信功能 和服务能力各不相同, 需要提出对应的评估指标要 求。例如, 针对军事对抗的卫星通信, 更为注重稳 定性和安全性，在评估指标构建上应重点考虑抗电 磁干扰性能与抗摧毁威胁性能; 针对作战领域的卫 星通信, 考虑到海 / 陆 / 空 / 天多维度机动性、地域 性和信息可靠性，在评估指标构建上应关注覆盖范 围、信息防护、自适应能力等方面。针对 PNTRC 拟承担的通信服务一一克服地面通信网络覆盖范围 不足的局限、实现天地一体移动宽带通信传输, 应
着重考虑覆盖能力、通信容量、通信质量等通信性 能相关的指标, 并据此建立通信能力仿真评估指标 体系。

\section{（二）遥感仿真评估}

遥感卫星主要开展探测感知、持续监测类的任 务, 有着过境回传 (信息) 的特性。PNTRC 具有 的通信能力解决了遥感卫星的信息传输难点, 使得 针对遥感卫星的评估重点聚焦于遥感方面的能力, 仿真设计应重点考虑信息获取能力, 需要应用成像 采集模型。

卫星遥感涉及的技术内容广泛, 在遥感能力方 面存在着多样化的划分标准、多类型的评估方案。 例如, 针对遥感卫星的系统信息支援能力, 更为关 注对具体任务的匹配程度, 评估指标中服务有效时 长是关键内容。针对 PNTRC 遥感服务——快速遥 感全天候与全天时、实时获取光学和雷达视频数据, 可从遥感的单一能力出发, 主要采用目标识别率、 成像质量、单次工作时间等指标来构建遥感能力仿 真评估模型。

\section{（三）导航定位授时仿真评估}

卫星导航系统提供多样服务, 包含导航定位、 授时、信息保障、环境检测和气象保障等，存在多 样的性能规范及指标体系。例如, 美国全球定位系 统 (GPS)、俄罗斯全球卫星导航系统 (GLONASS)、 欧洲伽利略卫星导航系统 (GALILEO) 都有着各 自的标准。PNTRC 在功能方面结合了北斗卫星导 航系统（BDS）中的相关卫星，对其导航定位授时 的能力评估, 可以北斗系统能力为参考标准。

卫星导航定位授时的能力主要由定位精度、授 时精度、测速精度等指标构成, 多数评估研究都 依据功能指标来开展。例如, 参考 BDS、GPS 和 GLONASS 的服务性能指标, 进行对比分析后对多 项性能指标进行评估。针对 PNTRC 导航定位授时 服务一一实时定位精度达到米和分米级、提供高精 度实时导航信息、精密授时达到纳秒级、提供时间 信息和同步信息，应着重考虑以服务精度为主线的 评估需求，如空间分辨率、定位精度、水平保护级 (HPL) 精度等, 据此构建导航定位授时能力仿真 评估模型。 


\section{四、PNTRC 仿真评估关键技术}

\section{（一）场景可扩展的高效分布式仿真技术}

1. 仿真场景可扩展设计

PNTRC 用于支持和提供高性能的导航、定位、 授时、遥感、通信一体化实时服务。在 PNTRC 仿 真分析过程中, 针对不同业务的特点, 采用多种 仿真软件（如 OPNET [21]、Exata [22]）搭建仿真 场景; 接口协议可兼容多种仿真软件（如 STK 软 件), 在实现多种仿真软件匹配和兼容的同时，达 到仿真场景可扩展的设计目的。

\section{2. 面向大尺度、多场景的分布式仿真}

天基信息实时智能服务涉及卫星、信关站、用 户站、固定用户、移动用户等多种实体，星间、星 地链路处于复杂多变的环境中。如果仅采用单一仿 真软件开展场景设计、链路规划、业务部署和性能 分析输出, 将面临低效率、高耦合等突出问题。分 布式仿真将整体仿真任务按照组成结构的特征划分 为多个模块, 单个模块独立进行局部功能仿真; 通 过底层支持模块来进行跨模块的信息交互，由此协 同完成整体仿真任务。
PNTRC 仿真架构包括：网络仿真单元、信道 与链路仿真单元、效能评估单元、任务规划单元、 演示单元和控制台单元等 (见图 1), 通过 HAL/RTI 实现各个功能单元之间的互联互通。控制台单元通 过仿真参数配置, 实现一体化仿真过程的控制和配 置; 任务规划单元根据场景参数和业务参数进行路 径规划和计算输出规划, 并将结果文件发送到仿真 和演示单元; 网络仿真单元根据仿真参数生成仿真 配置文件，接受控制台指令启动仿真，在仿真过程 中实时发送统计指标到演示和评估单元; 信道与链 路仿真单元与 MATLAB 仿真软件 [23] 连接, 对信 道与链路进行仿真; 演示单元根据演示场景参数、 规划结果文件以及仿真进行时的输出指标进行场景 演示; 效能评估单元依据评估算法, 对导航增强、 在轨信息处理和网络通信的效能进行评估。

\section{（二）面向动态天基信息系统仿真的评估技术}

1. 多层次指标体系构建

PNTRC 功能具有扩展性: 单一任务可由多种 功能组合完成, 单一功能又可由系统的多种能力支 撑, 因此仿真评估采用的指标体系应是多层次的。
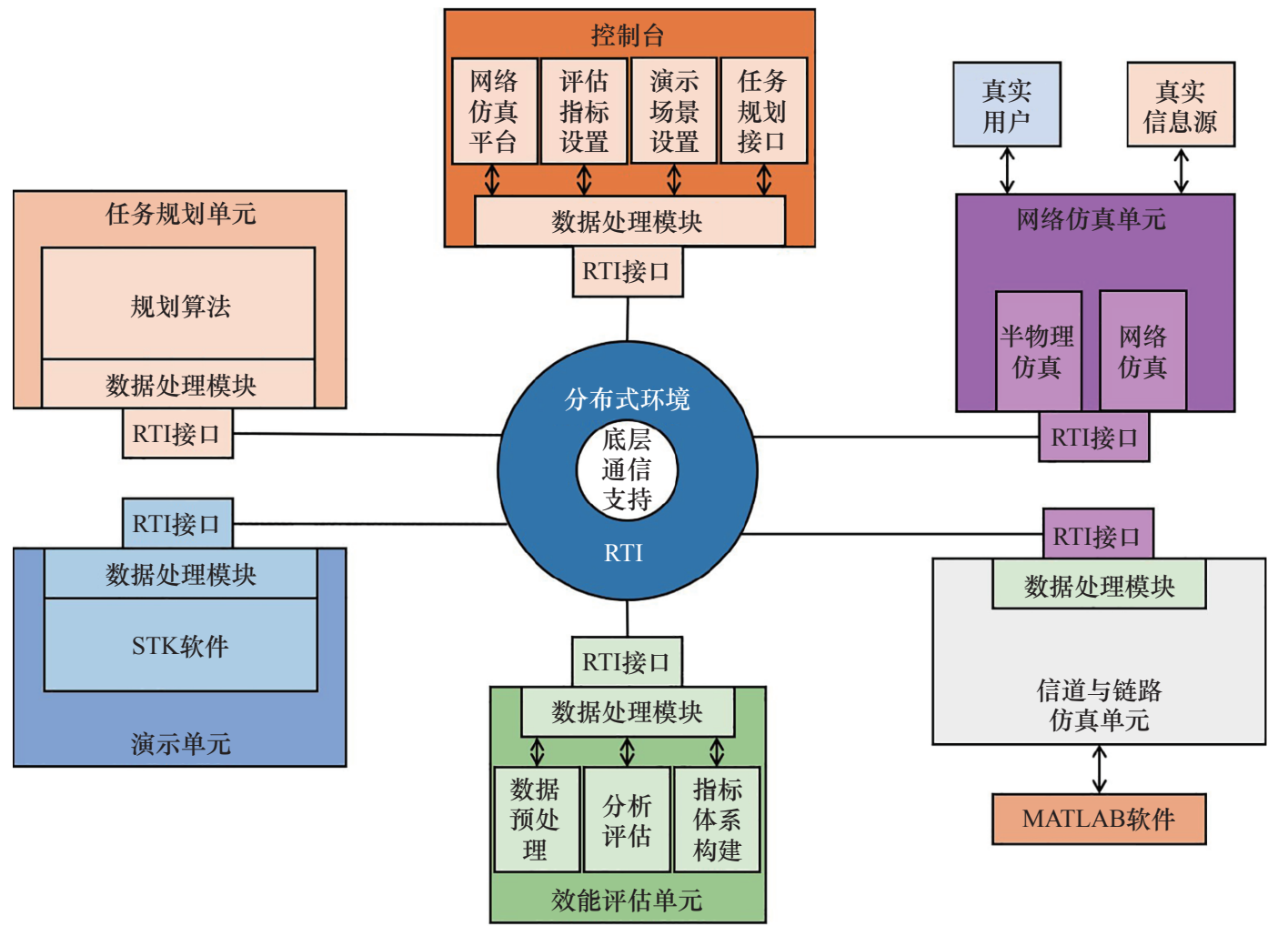

图 1 PNTRC 仿真功能单元构成图 
指标体系的选取应充分考虑系统各方面能力以实施 定量评价, 合理确定各项指标的重要性并选定指标 权重。通常采用多层级评价指标体系: 依据评估需 求, 合理分解目标; 按因素间的关系划分层级, 使 得结构清晰。

\section{2. 基于时变图分析的新型评估技术}

（1）时变图模型构建与数据处理

PNTRC 仿真具有规模大、动态性、耗时长等 特点, 且指标数据是多维向量。针对仿真体系架构 与网络特征, 开展基于时变图理论的网络模型构建 与动态性能仿真数据算法设计; 在保留网络变化特 征的基础上，提取典型仿真数据作为评估样本。这 类算法可以表达新型评估系统特征, 具有复杂度低、 可直观展现网络时序变化等优点。

\section{（2）天基信息系统新型评估技术}

梳理现阶段主流评估方法的技术特点, 针对 PNTRC 应用特征和时变图特征的动态数据结构, 优化改进基于熵值 / 模糊模型等概念的算法设计思 路, 提出适用于动态变化仿真数据的新型评估算法 (涉及离差、模糊、组合、关联等)。这类新算法可 对系统仿真性能进行客观评估（基于数据的评估）, 同时不失可解释性（基于经验的权重处理），在动 态评估时变图数据方面具有良好的效能。此外, 新 算法具有较低的时间和空间复杂度, 通过参数配置 即可满足同一场景下多次开展仿真数据评估的实际 需求。

（3）指标的体系贡献率

体系贡献率表征复杂系统输出性能不确定性向 各个输入变量的分配情况, 可为系统的可靠性设计、 评估模型的合理简化提供依据。基于随机森林的体 系贡献率算法，利用输入变量（指标信息）的分布 范围及其波动情况等信息, 衡量输入变量的不确定 性对于网络性能贡献程度。应用这一算法, 可针对 性地优化网络结构, 提高对 PNTRC 仿真结果进行 评估的综合效能。

\section{（三）PNTRC 仿真系统建模}

\section{1. 基于仿真数据驱动的天基信息网络建模}

天基信息网络仿真思路为：(1)构建局部系统模 型, 按照结构和功能进行组合, 搭建整体仿真系统; (2)依据 PNTRC 实际系统状态, 设计仿真实验参数; (3)通过仿真平台实施 PNTRC 工作过程模拟，针对
不同的设计参数和运行目标进行多次仿真; (4)通过 多次仿真, 获取有关仿真系统不确定性的数据, 输 出相应的统计指标; (5)以仿真统计输出数据和网络 设计参数为驱动, 对空间信息网络开展全局性的建 模分析。

仿真数据驱动的建模过程主要包括：(1)基于 目标需求的设计要素提取, 如业务接入带宽、通 信路由方式等; (2)依托仿真平台, 如 OPNET 进行 参数设计, 完成仿真场景搭建; (3)通过统计场景 实时仿真数据, 有效提取相应系统能力下的性能指 标; (4)采用新型评估算法对性能指标进行评估，依 据评估结果来改进要素提取、优化参数设计、完善 PNTRC 仿真模型。

2. 面向动态信息的天基信息网络建模

PNTRC 的重要特征是具有高动态性, 即天 基信息网络的拓扑结构时刻处于动态变化中。网 络时空复杂但又有一定规律：具有特定的空间构 型、时间维度呈现出周期性和节律性，还具有 “多 网联动” “多域铰链” 的特性。这些特征在进行 PNTRC 建模时应予以综合、妥善的考虑。本文基 于广义混合概率过程（HPP）模型和模型权重退化 上界 (WDUCB) 获取准则, 提出了一种多模态异 构网络模型表示与编排的贝叶斯优化方法, 以匹 配空间异构网络动态多业务场景的需求。仿真应 用结果表明该方法在异构动态网络表示和优化方 面具有良好的性能。

\section{五、面向 PNTRC 遥感业务的仿真评估应用}

采用天基信息实时智能服务仿真评估系统，针 对 PNTRC 遥感业务开展仿真分析及效能评估。作 为理论研究, 选取国内外具有代表性的低轨通信星 座与 PNTRC 遥感星座进行组网服务：中国航天科 工集团有限公司拟建 “虹云” 网络，中国航天科技 集团有限公司拟建 “鸿赝” 网络，美国一网公司 (OneWeb) 拟建 OneWeb 网络。通过仿真获得 3 种 组网服务的性能对照, 为未来构建更为优化的空间 信息网络架构提供设计参考。

\section{（一）遥感星座与低轨通信星座概况}

跨星座组网服务的优势在于, 利用低轨通信星 座的轨道高度优势, 将 PNTRC 遥感数据即时发送 
到更广阔的地面区域, 远期将覆盖全球范围。这是 PNTRC 遥感星座无法独立实现的能力。应用仿真 效能评估系统，对比了 “虹云” “鸿雁” 以及 OneWeb 3 种网络分别与 PNTRC 遥感星座进行组网的 服务性能。

PNTRC 遥感星座的设计轨道高度为 $500 \mathrm{~km}$, 总共 15 个轨道面, 每个轨道面上分布 6 颗卫星, 随机选取其中 10 颗卫星用于仿真实验。设计有 11 个地面站（均位于境内）, 包括: 北京、成都、 福州、广州、贵阳、哈尔滨、呼和浩特、济南、乌 鲁木齐、武汉、西宁。在全部地面站都开展相关业 务的条件下，“虹云” 网络在运行期间能够同时提供 接入服务的卫星数量平均为 11 颗, “鸿雁” 网络平 均为 8 颗, OneWeb 网络平均为 12 颗。

\section{（二）PNTRC 遥感业务传输性能分析}

针对 PNTRC 遥感业务传输性能评估需求, 重 点选取遥感视频业务丢包率与时延, 遥感图像业务 丢包率与时延等关键指标进行分析。

在传输遥感视频业务时, OneWeb 的丢包性 能和时延均优于 “虹云” “鸿雁” 网络（见图 2 和 图 3), 这是因为 OneWeb 的星座覆盖范围更广。然 而, OneWeb 覆盖更为密集, 其单个卫星的波束角 更小，存在着更多数量的波束切换过程。在视频业 务流增多的工况下，由于视频流传输持续的时间一 般相对于其他业务较长，频繁的波束切换将导致时 延在一定程度上的增加现象。

在传输遥感图像业务时, OneWeb 覆盖更为密

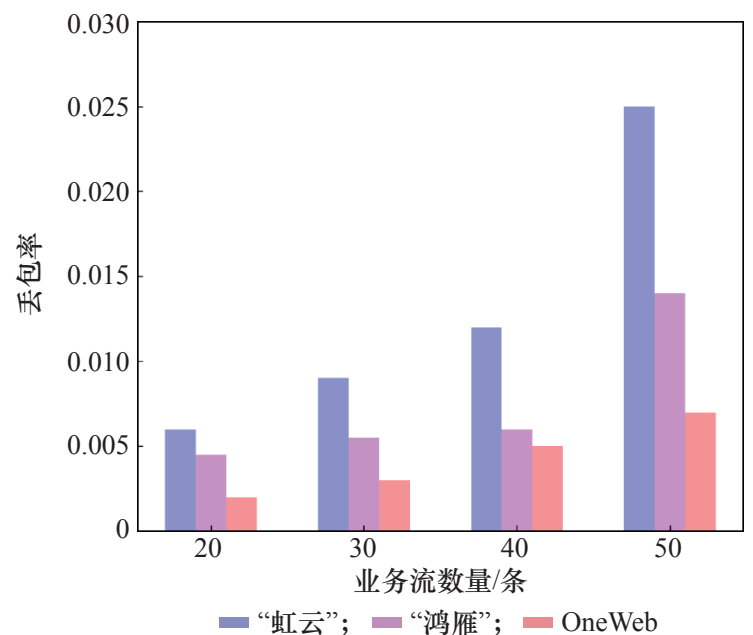

图 2 遥感视频业务丢包率对比
集，可用卫星数量也更多，因而丢包性能和时延均 优于 “虹云” “鸿雁” 网络（见图 4 和图 5), 即表 现出更高的容量和稳定性。另外, 遥感图像传输业 务的持续时间一般比较短, 而瞬时流量比较大, 传

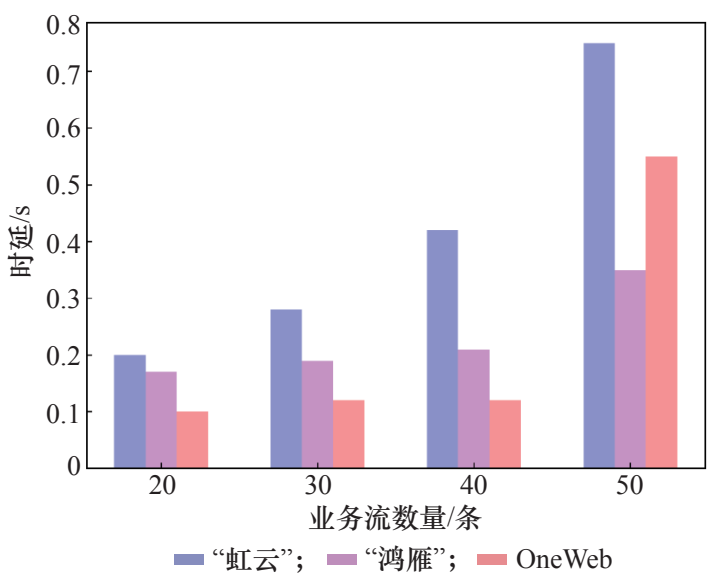

图 3 遥感视频业务时延对比

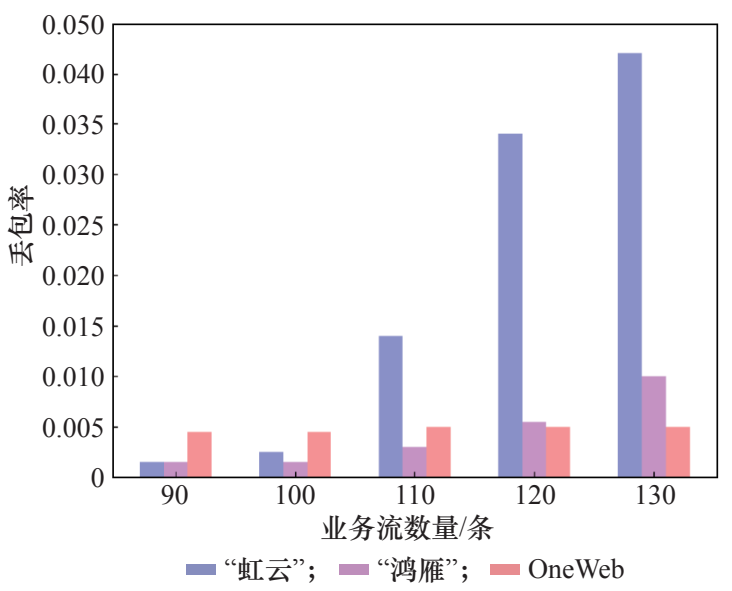

图 4 遥感图像业务丢包率对比

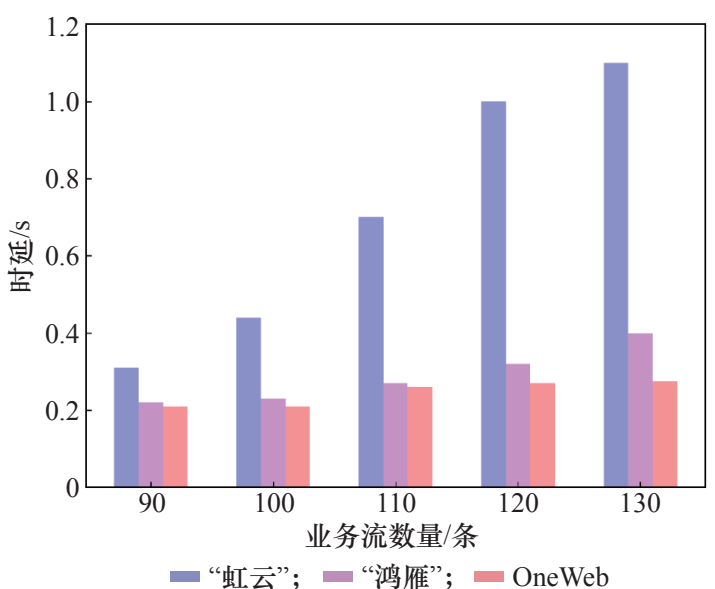

图 5 遥感图像业务时延对比 
输过程中频繁的波束切换对于业务性能影响较小。

\section{（三）低轨通信卫星单星通信容量分析}

对于遥感视频业务和遥感图像业务, 根据上文 的遥感业务可用条数, 分别计算出 PNTRC 遥感星 座的单星业务容量（见图 6 和图 7)。

随着组网星座中卫星数量的增多, 遥感视频和 遥感图像业务的总业务容量也随之提升，但提升幅 度不是线性的: “虹云” 的单星容量高于 “鸿雁” 和 OneWeb。着眼未来 PNTRC 遥感业务全球覆盖的能 力需求, 低轨通信星座的单颗卫星覆盖服务能力仍 需进一步提高。

\section{六、结语}

针对 PNTRC 论证和建设需求, 本文在梳理 空间信息系统仿真评估研究现状的基础上，对

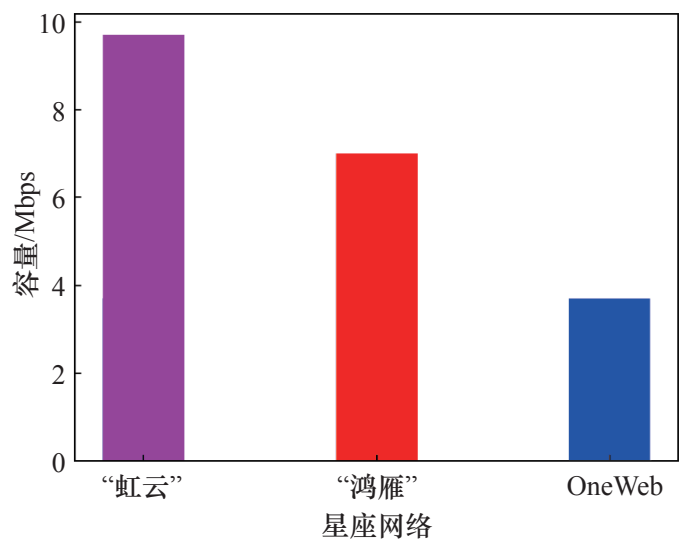

图 6 遥感视频业务单星容量对比

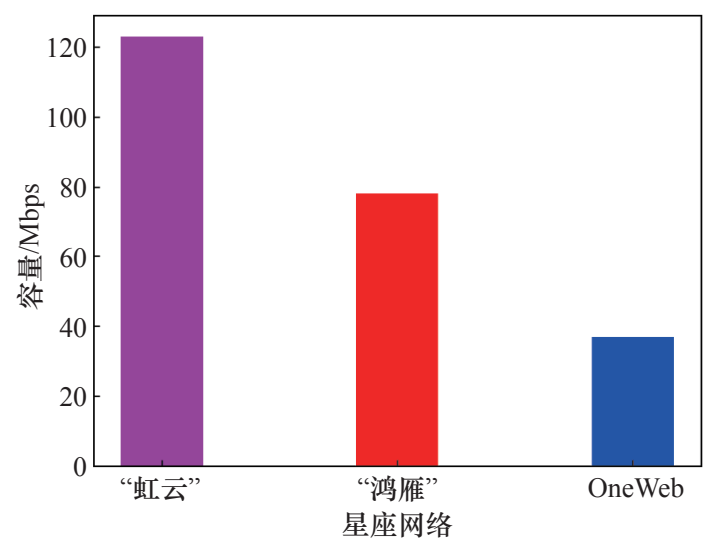

图 7 遥感图像业务单星容量对比
PNTRC 通信、遥感、导航定位授时等业务的总体 特征等进行了分析, 重点阐述了 PNTRC 仿真评估 涉及的高效分布式仿真、动态系统仿真、仿真系统 建模等关键技术。基于发展的仿真评估系统, 面向 PNTRC 遥感业务完成了应用研究, 获得了与 “虹 云” “鸿雁” 和 OneWeb 3 种网络进行组网服务的性 能对照, 为我国构建更为优化的空间信息网络架构 提供了设计参考。

PNTRC 的通信 / 导航 / 遥感业务一体化, 易 于实现军民功能结合, 对保障国家安全、经济社会 发展具有重大潜在价值, 而仿真评估是加强和优化 PNTRC 顶层设计的关键内容。基于仿真大数据驱 动的网络建模和网络要素的发现, 必将为 PNTRC 优化和建设提供有力支撑, 而仿真评估研究也将朝 着分布式仿真、时空动态分析评估、体系化建模、 模型泛化等方向深入发展。

\section{参考文献}

[1] 李德仁. 论军民深度融合的通导遥一体化空天信息实时智能服 务系统 [J]. 军民两用技术与产品, 2018 (15): 14-17.

Li D R. On the real-time intelligent service system integrating communication, navigation, remote sensing for military and civilian [J]. Dual Use Technologies \& Products, 2018 (15): 14-17.

[2] 李德仁, 沈欣, 李迪龙, 等. 论军民融合的卫星通信、遥感、导航 一体天基信息实时服务系统 [J]. 武汉大学学报(信息科学版), 2017, 42(11): 1501-1505.

Li D R, Shen X, Li D L, et al. On civil-military integrated spacebased real-time information service system [J]. Geomatics and Information Science of Wuhan University, 2017, 42(11): 15011505 .

[3] Luu K, Martin M, Stallard M, et al. University nanosatellite distributed satellite capabilities to support TechSat 21 [R]. Logan: American Institute of Aeronautics and Astronautics / Utah State University, 1999

[4] Bertiger W, Bar-Server Y, Battadpur S, et al. GRACE: Millineters and microns in orbit [R]. Washington DC: National Aeronautics and Space Administration, 2002.

[5] Zencik R, Kohlhepp K. GPS micro navigation and communication system for clusters of micro and nanosatellites [R]. Big Sky: Institute of Electrical and Electronics Engineers / Utah State University, 2000

[6] Gunnam K K, Hughes D C, Junkins J L, et al. A vision-based DSP embedded navigation sensor [J]. IEEE Sensors Journal, 2002, 2(5): 428-442.

[7] Tien J Y, Srinivasan J M, Young L E, et al. Formation acquisition sensor for the Terrestrial Planet Finder (TPF) mission [R]. Big Sky: Institute of Electrical and Electronics Engineers / Utah State University, 2004

[8] Purcell G, Kuang D, Lichten S, et al. Autonomous formation 
flyer (AFF) sensor technology development [R]. Washington DC: National Aeronautics and Space Administration, 1998.

[9] Gill E, Steckling M, Butz P. Gemini: A mileston towards autonomous formation flying [R]. Noordwijk: European Space Agency, 2001.

[10] 王航, 陈勇, 宋旭民, 等. 基于DDS的空间平台仿真试验床信息 交互技术研究 [J]. 现代电子技术, 2014, 37(20): 7-10.

Wang H, Chen Y, Song X M, et al. Research on informationexchange technology for space simulative test-bed based on DDS [J]. Modern Electronics Technique, 2014, 37(20): 7-10.

[11] 高小亮, 马骏骁, 李劼. HLA-DDS分布式通信卫星仿真系统 [J]. 国外电子测量技术, 2017, 36(6): 89-95.

Gao X L, Ma J X, Li J. HLA-DDS distributed simulation system of telecommunication satellite $[\mathrm{J}]$. Foreign Electronic Measurement Technology, 2017, 36(6): 89-95.

[12] 张志鹤, 史璐莎, 张斌, 等. 一种基于DDS与HLA的实时性联合 仿真系统 [J]. 电子设计工程, 2017, 25(10): 26-30.

Zhang Z H, Shi L S, Zhang B, et al. A real-time simulation system based on DDS \& HLA [J]. Electronic Design Engineering, 2017, 25(10):26-30.

[13] 鲁娜, 张杰, 马东堂. 卫星通信系统抗干扰性能评估指标体系研 究 [J]. 现代电子技术, 2014, 37(19): 29-32.

Lu N, Zhang J, Ma D T. Study on anti-jamming performance evaluation index system of satellite communication system [J]. Modern Electronics Technique, 2014, 37(19): 29-32.

[14] 许相莉, 胡晓峰, 秦永刚. 基于“四域”的卫星通信系统效能评估 指标体系 [J]. 指挥与控制学报, 2015, 1(2): 220-222.

Xu X L, Hu X F, Qin Y G. A type of effectiveness evaluation assessment indicator system for satellite communication system based on the "Four-Domain" [J]. Journal of Command and Control, 2015, 1(2): 220-222.

[15] 彭耿. 面向任务的遥感卫星信息支援能力评估指标体系构建 [J]. 指挥控制与仿真, 2019, 41(2): 15-19.

Peng G. Index system construction of information support capability evaluation of remote sensing satellite task-oriented [J]. Command Control \& Simulation, 2019, 41(2): 15-19.

[16] 覃鹏程, 郝胜勇, 秦国政. 基于遥感卫星应用体系贡献度评估方
法研究 [J]. 电子设计工程, 2019, 27(2):70-73, 79.

Qin P C, Hao S Y, Qin G Z. Research on evaluation method of contribution rate to SOS for remote sensing satellite application [J]. Electronic Design Engineering, 2019, 27(2): 70-73, 79.

[17] 段亚军, 武昌, 李成恩. 一种改进的卫星导航系统效能评估模型 [J]. 火力与指挥控制, 2008, 33(5): 133-136.

Duan Y J, Wu C, Li C E. An improved model of effectiveness evaluation of satellite navigation system [J]. Fire Control \& Command Control, 2008, 33(5): 133-136.

[18] 郭树人, 蔡洪亮, 孟轶男, 等. 北斗三号导航定位技术体制与服 务性能 [J]. 测绘学报, 2019, 48(7): 810-821.

Guo S R, Cai H L, Meng Y N, et al. BDS-3 RNSS technical characteristics and service performance [J]. Acta Geodaetica et Cartographica Sinica, 2019, 48(7): 810-821.

[19] 李张元, 赵忠文, 杨苗本. 基于复杂网络的空间信息网脆弱性研 究综述 [J]. 兵器装备工程学报, 2018, 39(6): 159-164.

Li Z Y, Zhao Z W, Yang M B. Literature review on vulnerability of space information network based on complex networks [J]. Journal of Ordnance Equipment Engineering, 2018, 39(6): 159-164.

[20] 杨兴, 吴静, 江昊, 等. 基于仿真数据驱动的空间信息网络建模 方法 [J]. 系统仿真学报, 2018, 30(11): 4323-4333, 4339.

Yang X, Wu J, Jiang H, et al. Simulation data-driven modeling approach for space information network [J]. Journal of System Simulation, 2018, 30(11): 4323-4333, 4339.

[21] 郜林. 基于OPNET的通信网仿真 [M]. 西安: 西安电子科技大学 出版社, 2018.

Gao L. Communication network simulation based on OPNET [M]. Xi'an: Xidian University Press, 2018.

[22] 徐以标, 王兴建, 尹建月. 基于Exata的网络通信协议仿真 [J]. 计 算机与数字工程, 2014 (11): 2212-2216.

Xu Y B, Wang X J, Yin J Y. Simulation of network communication protocols based on Exata [J]. Computer and Digital Engineering, 2014 (11): 2212-2216.

[23] Proakis J G, Salehi M, Bauch G. Modern communication systems using MATLAB [M]. Noida, Uttar Pradesh: Cengage Learning India Private Limited, 2013. 